\title{
Correlation Dynamics of Quantum Fields and Black Hole Information Paradox *
}

\author{
B. L. $\mathrm{Hu}^{\dagger}$ \\ Department of Physics, University of Maryland, College Park, MD 20742, USA
}

(umdpp 96-51, November 15, 1995)

\begin{abstract}
In recent years a statistical mechanics description of particles, fields and spacetime based on the concept of quantum open systems and the influence functional formalism has been introduced. It reproduces in full the established theory of quantum fields in curved spacetime and contains also a microscopic description of their statistical properties, such as noise, fluctuations, decoherence, and dissipation. This new framework allows one to explore the quantum statistical properties of spacetime at the interface between the semiclassical and quantum gravity regimes, as well as important non-equilibrium processes in the early universe and black holes, such as particle creation, entropy generation, galaxy formation, Hawking radiation, gravitational collapse, backreaction and the black hole end-state and information lost issues. Here we give a summary of the theory of correlation dynamics of quantum fields and describe how this conceptual scheme coupled with scaling behavior near the infrared limit can shed light on the black hole information paradox.
\end{abstract}

*Lectures given at the International School of Astrophysics "D. Chalonge": String Gravity and Physics at the Planck Energy Scale, Erice, Sept. 1995. Proceedings edited by N. Sanchez, (World Scientific, Singapore, 1996)

†Email: hu@umdhep.umd.edu 


\section{Stochastic Theory of Quantum Fields and Spacetime}

I gave two lectures at this School. Since they contain material already published, I do not want to repeat them here. Instead, I will mention some on-going projects in our research program and discuss some ideas on how the correlations of quantum fields can be used to address the black hole information paradox issue. The abstracts of these two lectures, and relevant references (where the background material and bibliography can be found) are given below. (For a more comprehensive recent review, see [1])

\section{Lecture 1. Stochastic Analysis of Particles and Fields: The Effec- tive Action and the Influence Functional Methods}

We first summarize the in-out (Schwinger-DeWitt) effective action formalism [2] in quantum field theory in curved spacetime [3] and point out the need for generalization to the in-in [4] (Schwinger-Keldysh, or closed-time-path CTP) [5, 6, 7] formalism to treat particle creation and backreaction problems in semiclassical gravity [8]. We then discuss the statistical mechanics of quantum open systems by way of the Brownian motion model, using the influence functional (IF) [9] formalism of Feynman-Vernon [10, 11]. By viewing the subjects of interest in semiclassical gravity as open systems, where the classical geometry is treated as the system and the quantum matter field as the environment, and using the close relation of the CTP and the IF methods, we can examine both the quantum and the statistical mechanical attributes of the processes involved. We derive an expression for the CTP effective action or the IF in terms of the Bogolubov coefficients relating the second quantized operators between two Fock spaces of the field theory [12, 13], thus connecting back with the established theories of quantum fields in curved spacetimes. As example, we show how one can derive the Hawking-Unruh radiations [14, 15] from a non-equilibrium statistical mechanics (NESM) viewpoint [12]. These effects are understood as resulting from the scaling or amplification of quantum noise between observers in different kinematic or dynamical states [16, 17]. Viewing these effects in this light would enable one to consider fully non-equilibrium processes not easily approachable by conventional (e.g., geometric or thermodynamics) means.

\section{Lecture 2. Einstein-Langevin Equation in Semiclassical Gravity: Backreaction as Fluctuation-Dissipation Relation}

The influence functional method in NESM (which includes the CTP effective action method in quantum field theory) explicates the meaning and the interrelation [18, 1] of the many quantum processes involved in the backreaction problem, such as particle creation, noise, fluctuations [13], decoherence [19, 20] and dissipation [8]. The backreaction of created particles and their fluctuations is described by an equation of motion derivable from the influence action in the form of an Einstein-Langevin equation [21]. It contains a dissipative term for the dynamics of spacetime and a noise term related to the fluctuations of particle creation in the matter field. As examples, we first study the case of a free quantum scalar field in a 
spatially flat Friedmann- Robertson-Walker universe and derive the Einstein-Langevin equations for the scale factor for these semiclassical cosmologies [13]. Then using the well-studied model of a quantum scalar field in a Bianchi Type-I universe we illustrate how this Langevin equation and the noise term are derived and show how the creation of particles and the dissipation of anisotropy during the expansion of the very early universe can be understood as the manifestation of a fluctuation-dissipation relation [22]. This theorem, which exists under very general conditions for dissipations in the dynamics of a system, and the noise and fluctuations in its environment, embodies the backreaction effect of matter fields on the spacetime.

Our approach based on statistical field theory extends the conventional theory of semiclassical gravity based on a semiclassical Einstein equation with a source given by the expectation value of the energy-momentum tensor, to that based on a Langevin-type equation, where the dynamics and fluctuations of spacetime are driven by quantum fluctuations of the matter field. This generalized framework is useful for the investigation of quantum processes in the early universe involving fluctuations, vacuum stability and phase transition phenomena and the non-equilibrium statistical mechanics of black holes. It is also essential to an understanding of the cross-over behavior between quantum gravity and general relativity.

\section{Related problems}

Let me also mention some related problems currently under investigation:

1) Metric Fluctuations in Semiclassical Gravity [21, 22, 23]

2) Quantum Fluctuations and Structure Formation in the early universe [24, 13, 25]

3) Correlation, Decoherence, Dissipation and Noise of Quantum Fields [26, 27]

4) Stochastic Theory of Accelerated Detectors [28, 29]

5) Backreaction of Unruh Radiation on an Accelerated Detector and a Moving Mirror [30, 31]

6) Fluctuation-Dissipation Relation for a Radiating Black Hole [32]

The first round of attack on Problems 1) to 4) have been completed recently. Problems 5) and 6) are in progress. In the following I will briefly discuss how correlation dynamics in quantum fields might play a role in the black hole information paradox problem. Sec. 2 contains a summary from [27]. Sec. 3 contains entirely new material. The reader should nevertheless be warned that this investigation is still in a preliminary stage, and the ideas are largely speculative. Also since this is more in the nature of a progress report than a review, I have not attempted to include a complete bibliography. Other related work can be found from the reference lists of the quoted papers.

\section{Statistical Mechanics of an Interacting Quantum Field}

We begin with an analysis of the statistical mechanics of an interacting quantum field. This familiar subject which one learns in the first lessons of quantum field theory is surprisingly 
rich in its statistical mechanics content, much like the role an ordinary box of gas molecules plays in Boltzmann's sophisticated theory of kinetics and dissipation.

\subsection{Correlation, noise, and decoherence in quantum fields}

In two recent papers [26, 27] Calzetta and I studied the statistical mechanical properties of interacting quantum fields in terms of the dynamics of the correlation functions. We started from the thesis that the full dynamics of an interacting quantum field may be described by means of the Dyson- Schwinger equations governing the infinite hierarchy of Wightman functions which measure the correlations of the field. We showed how this hierarchy of equations can be obtained from the variation of the infinite particle irreducible, or 'master' effective action (MEA). Truncation of this hierarchy gives rise to a quantum subdynamics governing a finite number of correlation functions (which constitute the 'system'), and expression of the higher order correlation functions (which constitute the 'environment') in terms of the lower-order ones by functional relations ('slaving' or 'factorization') induces dissipation in the dynamics of the subsystem driven by the stochastic fluctuations of the environment, which we call the 'correlation noises'. These two aspects are related by the fluctuation-dissipation relation. This is the quantum field equivalent of the BBGKY hierarchy in Boltzmann's theory. Any subsystem involving a finite number of correlation functions defines an effective theory, which is, by this reasoning, intrinsically dissipative. The relation of loop expansion and correlation order is expounded. We see that ordinary quantum field theory which involves only the mean field and a two-point function, or any finite-loop effective action in a perturbative theory are, by nature, effective theories which possess these properties. Histories defined by lower-order correlation functions can be decohered by the noises from the higher order functions and acquire classical stochastic attributes. We think this scheme invoking the correlation order is a natural way to describe the quantum to classical transition for a closed system as it avoids ad hoc stipulation of the system-environment split. It is through decoherence that the subsystem variables become classical and the subdynamics becomes stochastic.

Our viewpoint here is motivated by classical kinetic theory. In the dynamics of a dilute gas [33, 34, 35] the exact Newton's or Hamilton's equations for the evolution of a many body system may be translated into a Liouville equation for the distribution function or the BBGKY hierarchy for the sequence of partial (n- particle) distributions. This reformulation is only formal, which involves no loss of information or predictability. Physical description of the dynamics comes from truncating the BBGKY hierarchy and introducing a factorization condition like the molecular chaos assumption, where the higher order distributions are substituted by functionals of the lower order. Constructed perturbatively, this effective theory follows only approximately the actual dynamics. Moreover, these functionals embody some relevant boundary conditions (such as the 'weakening of correlations' hypothesis [33]), which make them noninvariant upon time reversal. This is how dissipation in the explicitly irreversible Boltzmann's equation appears. Đ.

\footnotetext{
${ }^{1}$ On closer examination, it is seen that the one- particle distribution function itself describes only the
} 
Correlation dynamics described by the Dyson-Schwinger hierarchy derived from the master effective action: Truncation and Factorization

We want to describe a quantum field in terms of the mean field and the (infinite number of) correlation functions. Here, different from the conventional treatment, we view the 'mean' field not as the actual expectation value of the field, but rather as representing the local value of the field within one particular history. Quantum evolution encompasses the coherent superposition of all possible histories [19] and these quantities are subject to fluctuations. This naturally introduces into the theory stochastic elements, which has hitherto been ignored in the usual description of quantum field theory. The theory may be enlarged by including some correlation functions as independent variables along with the 'mean' field, which themselves are subject to fluctuations.

Our starting point is the well-known fact that the set of all Wightman functions (time ordered products of field operators) determines completely the quantum state of a field [38]. Instead of following the evolution of the field in any of the conventional representations (Schrödinger, Heisenberg or Dirac's), we focus on the dynamics of the full hierarchy of Wightman functions. To this end it is convenient to adopt Schwinger's "closed time-path" techniques [4], and consider time ordered Green functions as a subset of all Green functions path- ordered along a closed time loop. The dynamics of this larger set is described by the Dyson - Schwinger equations.

We first showed that the Dyson- Schwinger hierarchy may be obtained via the variational principle from a functional which we call the 'Master Effective Action' (MEA). This is a formal action functional where each Wightman function enters as an independent variable. We then showed that any field theory based on a finite number of (mean field plus ) correlation functions can be viewed as a subdynamics of the Dyson- Schwinger hierarchy. The specification of a subdynamics involves two steps: First, the hierarchy is truncated at a certain order. A finite set of variables, say, the lowest nth order correlation functions, is identified to be the 'relevant' [37] variables, which constitute the subsystem. Second, the remaining 'irrelevant' or 'environment' variables, say, the $\mathrm{n}+1$ to $\infty$ order correlation functions are slaved to the former. Slaving (or 'factorization' in the Boltzmann theory) means that irrelevant variables are substituted by set functionals of the relevant variables. The process of extraction of a subdynamics from the Dyson- Schwinger hierarchy has a correlate at the level of the effective action, where the MEA is truncated to a functional of a finite number of variables. The finite effective actions so obtained (the influence action [9]) are generally nonlocal and complex, which is what gives rise to the noise and dissipation in the subdynamics. Moreover, since the slaving process generally involves the choice of an arrow of time, it leads to irreversibility in

mean number of particles within a certain location in phase space; the actual number is also subject to fluctuations. From the average size of the equilibrium fluctuations, which can be determined from Einstein's formula, and the dissipative element of the dynamics, which is contained in the collision integral, it is possible to compute the stochastic driving force consistent with the fluctuation- dissipation relation near equilibrium 36 
the cloak of dissipation in the subdynamics [37.

\section{Decoherence of correlation history and correlation noise; fluctuations and dissipation}

Under realistic conditions, one may not be as much concerned with the full quantum evolution of the field as with the development of 'classical' theories where fields are described as c-numbers, plus perhaps a small number of correlation functions to keep track of fluctuations. These classical theories represent the physically observable dynamics after the process of decoherence [19, 20] has destroyed or diminished the coherence of the field. In any case, no actual observation could disclose the infinite number of degrees of freedom of the quantum field, and therefore any conceivable observational situation may be described in the language of a suitable complex 'classical' theory in this sense.

Decoherence is brought by the effect of a coarse-grained environment (or 'irrelevant' sector) on the system (or the 'relevant' sector). In simple models, this split is imposed by hand, as when some of the fields, or the field values within a certain region of spacetime, are chosen as relevant. Here, we will follow the approach of our earlier work on the decoherence of correlation histories [26]. There is no need to select a priori a relevant sector within the theory. Instead, we shall seek a natural criterium for successive truncations in the hierarchy of correlations, the degree of truncation depending on the stipulated accuracy of measurement which can be carried out on the system. In this framework, decoherence occurs as a consequence of the fluctuations in the higher order correlations and results in a classical dissipative dynamics of the lower order correlations.

Here, we adopt the consistent histories formulation of quantum mechanics [19] for the study of the quantum to classical transition problem. We consider the full evolution of the field described by the Dyson- Schwinger hierarchy as a fine- grained history while histories where only a finite number of Wightman functions are freely specified (with all others slaved to them) are therefore coarse- grained. We have shown that the finite effective actions obtained for the subsystems of lower-order correlations are related to the decoherence functional between two such histories of correlations [26], its acquiring an imaginary part signifies the existence of noise which facilitates decoherence. Thus decoherence of correlation histories is a necessary condition for the relevance of the c-number theory as a description of observable phenomena. It can be seen that if the c-number theory which emerges from the quantum subdynamics is dissipative, then it must also be stochastic. I From our correlation history viewpoint, the stochasticity is in fact not confined to the field distributions - the correlation functions would become stochastic as well [36.

Following Feynman and Vernon [9] in their illustration of how noise can be defined from

\footnotetext{
${ }^{2}$ Because the fundamental variables are quantum in nature, and therefore subject to fluctuations, a classical, dissipative dynamics would demand the accompaniment of stochastic sources (in agreement with the 'fluctuation - dissipation theorems', for, otherwise, the theory would permit unphysical phenomena as the damping away of zero - point fluctuations.) Of course, these uncontrollable fluctuations may be seen at the origin of many phenomena where structure seems to spring 'out of nothing', such as the nucleation of inhomogeneous true vacuum bubbles in a supercooled false vacuum, or the development of inhomogeneities out of a homogeneous early universe.
} 
the influence functional, we can relate the imaginary part of the finite effective actions describing the truncated correlations to the auto-correlation of the stochastic sources, i.e., correlation noises, which drive the c-number fields and their correlation functions via the Langevin- type equations. From the properties of the complete (unitary) field theory which constitutes the closed (untruncated) system, one can show that the imaginary part of the effective action is related to the nonlocal part of the real part of the effective action which depicts dissipation. This is where the fluctuation- dissipation theorem for non-equilibrium systems originates [22].

We thus see once again the intimate connection amongst the three aspects of the theory, decoherence, dissipation, and fluctuations [18, 39], now manifesting in the hierarchy of correlations which defines the subsystems.

\section{Correlations in a Quantum Field and Black Hole In- formation Paradox}

We view the black hole and the quantum field with Hawking radiation as a closed system. Even though the quantum field might be assumed to be free in the beginning, interaction still exists in its coupling with the black hole, especially when strong backreaction is included. We can model this complete system by an interacting quantum field. A particle- field system is a particular case of it. Of course a black hole is different from a particle. In this modeling, we will first explain how information is registered or 'lost' in an interacting quantum field, then the distinct features of a black hole and finally, the information loss paradox of black hole systems.

To approach the black hole information loss paradox we need to understand three conceptual points:

1) How does one characterize the information content of a quantum field (interacting, as a model for the black hole - quantum field closed system, with backreactions)

2) How does the information flow from one part of this closed system (hole) to another (field) and vice versa in the lifetime of the black hole? Does information really get 'lost'? If yes, where has it gone? Can it be retrieved? If no, where does it reside?

3) What is special about black hole radiation system as distinct from ordinary particle / field system?

The following is a brief sketch of the picture we have developed based on our studies of these issues in varying depths in the past ten years. The development of the correlation dynamics in quantum fields formalism was done with Esteban Calzetta [27], that of viewing black hole radiance and inflation as exponential scaling was explored with Yuhong Zhang 16, 40, 41 partly based on work on critical phenomena done earlier with Denjoe O'Connor 42. Studies of simple models to illuminate various points in this conceptual scheme are being pursued now with Alpan Raval. For general background on this issue, see the review 
of Page [43] which contains a comprehensive list of references till 1993, and Bekenstein [44]. For string theory related ideas, see, e.g., the recent reviews of 45. Our approach is closer in spirit to [46, 47.

\subsection{Correlation functions as registrar and correlation dynamics as flow-meter of information in quantum fields}

The set of correlation functions provides us with the means to register the information content of a quantum field. As mentioned above, the complete set of $n=\infty$ correlation (Wightman) functions carries the complete information about the quantum state of the field. A subset of it which defines the subsystem, such as the mean field and the 2-point function, as is used in the ordinary description of (effective) field theory, carries only partial information. The missing information resides in the correlation noise, and manifests as dissipation in the subsystem dynamics. In this framework, the entropy of an incompletely determined quantum system is simply given by $S=-\operatorname{Tr} \rho_{\text {red }} \ln \rho_{\text {red }}$, where the reduced density matrix of the subsystem (say, consisting of the lower correlation orders) is formed by integrating out the environmental variables (the higher correlation orders) after the hierarchy is truncated with factorization conditions.

While the set of correlation functions act as a registrar of information of the quantum system, keeping track of how much information resides in what order, the dynamics of correlations as depicted by the hierarchy of equations of motion derived from the master effective action depicts the flow of information from one order to another, up or down or criss-crossing the hierarchy. Correlation dynamics has been proposed for the description of many body systems before [34, and applied to molecular and plasma kinetics. In the light of the above theoretical description we see this scheme as a potentially powerful way to do quantum information systematics, i.e., keeping track of the content and flow of information in a coherent or partially coherent quantum system.

\subsection{Information appears lost to subsystems of lower order corre- lations - 'missing' information stored in higher order correla- tions}

Most measurements of a quantum field system are of a local or quasilocal nature. If one counts the information content of a system based on the mean field and the lowest order correlation functions, as in the conventional way (of defining quantum field theory in terms of, e.g., 2PI effective action), one would miss out a good portion of the information in the complete system, as much of that now resides in the higher order correlation functions in the hierarchy. These invoke nonlocal properties of the field, which are not easily accessible in the ordinary range of accuracy in measurements. Such an observer would then report on a loss of information in his way of accounting (which is taken to be in agreement with other observers with the same level of accuracy of measurement). Only observers which has access to all orders (the 'master' in the master effective action) would be able to see the 
complete development of the system and be able to tell when the nth order observer begins to lose track of the information count and reports an information loss. This is more easily seen in molecular dynamics: For observers confined to measuring one particle distribution functions (truncation of the BBGKY hierarchy) and with the molecular chaos assumptions implicitly invoked (factorization condition), he would report on information loss. This is how Boltzmann reasons out the appearance of dissipation in ordinary macroscopic physical phenomena. The same can be said about measurement of quantum systems.

\subsection{Exponential scaling in Hawking effect facilitates information transfer to the higher correlations. Black hole with its radia- tion contains full information, but retrieval requires probing the higher order nonlocal properties of the field}

How is this scheme useful in addressing the black hole information problem? How is the black hole / quantum field system different from the ordinary cases? The above scheme can explain the apparent loss of information in a quantum system, but there is an aspect distinct to black holes or systems emitting thermal radiance. Some years ago, I made the observation that all mechanisms of emission of (coherent) thermal radiance such as the Hawking effect in black holes, or the Unruh effect in accelerated detectors, involve an exponential redshifting process of or by the system. This can be compared to the scaling transformation in treating critical phenomena. After sufficient exponential redshifting (at late times of collapse) and the black hole is emitting thermal radiance, the system has reached a state equivalent to the approach to a critical point in phase transition. There, the physical properties of the system are dominated by the infrared behavior, and as such, the lowest order correlation functions are no longer sufficient to characterize the critical phenomena. The contribution of higher order correlation functions would become important. Note that in ordinary situations, only the mean field and the 2 or 3 point correlation functions are needed to give an adequate description of the dynamics of the system. But for black holes or similar systems where exponential red-shifting is at work, higher order correlations are readily activated. The information content profile for a quantum field in the presence of a black hole would be very different from ordinary systems, in that it is more heavily populated in the higher end of the spectrum (of correlation orders). If one carries out measurement at the lower end of the spectrum, one would erroneously conclude that there is information loss.

So, following the correlation dynamics of the black hole / field system, while the state of the combined system remains the same as it had begun, there is a continuous shifting of information content from the black hole to the higher correlations in the field as it evolves. Correlation dynamics of fields can be used to keep track of this information flow. We speculate that the information content of the field will be seen to shift from low correlation orders to the higher ones as Hawking radiation begins and continues. The end state of the system would have a black hole evaporated, and its information content transferred to the quantum field, with a significant portion of it residing in the higher order nonlocal correlations. 
This is, however, not the end of the story for the correlation dynamics and information flow in the field. The information contained in the field will continue, as it does in general situations, to shift across the hierarchy. As we know from the BBGKY description of molecular dynamics, after the higher correlation orders in the hierarchy have been populated and for systems subjected to exponential red-shifting this condition could be reached relatively quickly - the information will begin to trickle downwards in the hierarchy, though far slower than the other direction initially. The time it takes (with many criss-crossing) for the information to return to the original condition is the Poincare recurrence time. This time we suspect is the upper bound for the recoherence time, the time for a coherent quantum system interacting with some environment to regain its coherence [47]. It would be interesting to work out the information flow using the correlation dynamics scheme for a few sample systems, both classical and quantum, so as to distinguish the competing effects of different characteristic processes in these systems, some quantum, some statistical (e.g., decoherence time, relaxation time, recoherence time and recurrence time).

Our depiction above uses the interacting field model. Simpler cases might show somewhat degenerate behavior. f Details of these investigations will be reported in journal articles.

\section{Acknowledgement}

I thank the director of this School, Prof. Norma Sanchez, for expertly organizing a stimulating and enjoyable meeting. My lectures were based on work I did in the last two years with Esteban Calzetta, Andrew Matacz, Alpan Raval and Sukanya Sinha. Research is supported in part by the National Science Foundation under grant PHY94-21849.

\footnotetext{
${ }^{3}$ An example is the interesting result of recoherence reported by Anglin et al [47]. We think their reported result of a recoherence time of the order of the relaxation time is special to the simple model of particle free-field interaction. As the field modes couple only through their interaction with the particle, and not amongst themselves, there is no structure or dynamics of the information content of the field itself, and the only time scale for it to return is via interaction with the particle, which is why the recoherence time is related to the relaxation time of the particle. We expect in more general and complex systems (thus excluding many spin systems) the recoherence time is much longer than the relaxation time, more in the order of the recurrence time.
} 


\section{References}

[1] B. L. Hu, "Quantum Statistical Fields in Gravitation and Cosmology" in Proc. Third International Workshop on Thermal Field Theory and Applications, eds. R. Kobes and G. Kunstatter (World Scientific, Singapore, 1994) gr-qc/9403061

[2] J. Schwinger, Phys. Rev. 82, 664 (1951); B. S. DeWitt, Phys. Rep. 19C, 297 (1975)

[3] N. Birrell and P. W. C. Davies, Quantum Fields in Curved Spaces (Cambridge University Press, Cambridge, 1982)

[4] J. Schwinger, J. Math. Phys. 2 (1961) 407; P. M. Bakshi and K. T. Mahanthappa, J. Math. Phys. 4, 1 (1963), 4, 12 (1963). L. V. Keldysh, Zh. Eksp. Teor. Fiz. 47 , 1515 (1964) [Engl. trans. Sov. Phys. JEPT 20, 1018 (1965)]; G. Zhou, Z. Su, B. Hao and L. Yu, Phys. Rep. 118, 1 (1985); Z. Su, L. Y. Chen, X. Yu and K. Chou, Phys. Rev. B37, 9810 (1988); B. S. DeWitt, in Quantum Concepts in Space and Time ed. R. Penrose and C. J. Isham (Claredon Press, Oxford, 1986); R. D. Jordan, Phys. Rev. D33 , 44 (1986). E. Calzetta and B. L. Hu, Phys. Rev. D35, 495 (1987).

[5] E. Calzetta and B. L. Hu, Phys. Rev. D35, 495 (1987).

[6] E. Calzetta and B. L. Hu, Phys. Rev. D37, 2878 (1988)

[7] E. Calzetta and B. L. Hu, Phys. Rev. D40, 656 (1989).

[8] B. L. Hu, Physica A158, 399 (1989).

[9] R. Feynman and F. Vernon, Ann. Phys. (NY) 24, 118 (1963). R. Feynman and A. Hibbs, Quantum Mechanics and Path Integrals, (McGraw - Hill, New York, 1965). A. O. Caldeira and A. J. Leggett, Physica 121A, 587 (1983); Ann. Phys. (NY) 149, 374 (1983). H. Grabert, P. Schramm and G. L. Ingold, Phys. Rep. 168, 115 (1988). B. L. Hu, J. P. Paz and Y. Zhang, Phys. Rev. D45, 2843 (1992); D47, 1576 (1993)

[10] B. L. Hu, J. P. Paz and Y. Zhang, Phys. Rev. D45, 2843 (1992)

[11] B. L. Hu, J. P. Paz and Y. Zhang, Phys. Rev. D47, 1576 (1993)

[12] B. L. Hu and A. Matacz, Phys. Rev. D49, 6612 (1994).

[13] E. Calzetta and B. L. Hu, Phys. Rev D49, 6636 (1994).

[14] S.W. Hawking, Commun. Math. Phys. 43, 199 (1975).

[15] W.G. Unruh, Phys. Rev. D 14, 870 (1976). P. C. W. Davies, J. Phys. A: Gen. Phys. 8, 609 (1975). S. A. Fulling, Phys. Rev. D 7, 2850 (1973). 
[16] B. L. Hu, in Proceedings of the CAP-NSERC Summer Institute in Theoretical Physics, Vol 2 Edmonton, Canada, July 1987, eds K. Khanna, G. Kunstatter and H. Umezawa (World Scientific, Singapore, 1988)

[17] B. L. Hu, in Proceedings of the Fourth International Workshop on Thermal Field Theory Dalian, China, August 1995, eds Y. X. Gui and K. Khanna (World Scientific, Singapore, 1996)

[18] B. L. Hu, "Statistical Mechanics and Quantum Cosmology", in Proc. Second International Workshop on Thermal Fields and Their Applications, eds. H. Ezawa et al (North-Holland, Amsterdam, 1991).

[19] R. B. Griffiths, J. Stat. Phys. 36, 219 (1984); R. Omnés, J. Stat Phys. 53, 893, 933, 957 (1988); Ann. Phys. (N. Y.) 201, 354 (1990); Rev. Mod. Phys. 64, 339 (1992); The Interpretation of Quantum Mechanics, (Princeton University Press, Princeton (1994)). J. B. Hartle, "Quantum Mechanics of Closed Systems" in Directions in General Relativity Vol. 1, eds B. L. Hu, M. P. Ryan and C. V. Vishveswara (Cambridge Univ., Cambridge, 1993); M. Gell-Mann and J. B. Hartle, in Complexity, Entropy and the Physics of Information, ed. by W. H. Zurek (Addison-Wesley, Reading, 1990); J. B. Hartle and M. Gell- Mann, Phys. Rev. D47, 3345 (1993). J. P. Paz and S. Sinha, Phys. Rev. D44, 1038 (1991). H. F. Dowker and J. J. Halliwell, Phys. Rev. D46, 1580 (1992). T. Brun, Phys. Rev. D47, 3383 (1993). J. Twamley, Phys. Rev. D48, 5730 (1993). J. P. Paz and W. H. Zurek, Phys. Rev. D48, 2728 (1993).

[20] W. H. Zurek, Phys. Rev. D24, 1516 (1981); D26, 1862 (1982); in Frontiers of Nonequilibrium Statistical Physics, ed. G. T. Moore and M. O. Scully (Plenum, N. Y., 1986); Physics Today 44, 36 (1991); E. Joos and H. D. Zeh, Z. Phys. B59, 223 (1985); A. O. Caldeira and A. J. Leggett, Phys. Rev. A31, 1059 (1985); W. G. Unruh and W. H. Zurek, Phys. Rev. D40, 1071 (1989). B. L. Hu, J. P. Paz and Y. Zhang, Phys. Rev. D45, 2843 (1992); D47, 1576 (1993); J. P. Paz, S. Habib and W. H. Zurek, Phys. Rev. D47, 488 (1993). W. H. Zurek, J. P. Paz and S. Habib, Phys. Rev. Lett. 70, 1187 (1993); W. H. Zurek, Prog. Theor. Phys. 89, 281 (1993).

[21] B. L. Hu and A. Matacz, Phys. Rev. D51, 1577 (1995).

[22] B. L. Hu and S. Sinha, Phys. Rev. D51, 1587 (1995).

[23] A. Campos and E. Verdaguer, Phys. Rev. D53, (1996).

[24] B. L. Hu, J. P. Paz and Y. Zhang, "Quantum Origin of Noise and Fluctuation in Cosmology" in The Origin of Structure in the Universe Conference at Chateau du Pont d'Oye, Belgium, April, 1992, ed. E. Gunzig and P. Nardone (NATO ASI Series) (Plenum Press, New York, 1993) p. 227

[25] E. Calzetta and B. L. Hu, Phys. Rev. D52, (1995) 
[26] E. Calzetta and B. L. Hu, "Decoherence of Correlation Histories" in Directions in General Relativity, Vol II: Brill Festschrift, eds B. L. Hu and T. A. Jacobson (Cambridge University Press, Cambridge, 1993) gr-qc/9302013

[27] E. Calzetta and B. L. Hu, "Correlations, Decoherence, Disspation and Noise in Quantum Field Theory", in Heat Kernel Techniques and Quantum Gravity, ed. S. A. Fulling (Texas A\& M Press, College Station 1995). hep-th/9501040

[28] Alpan Raval, B. L. Hu and J. R. Anglin, "Stochastic Theory of Accelerated Detectors in a Quantum Field" (1995) gr-qc/9510002

[29] B. L. Hu, Alpan Raval, Don Koks and A. Matacz, "Near-Thermal Radiation in Detectors, Mirrors, Black Holes and Expanding Universe" (1995)

[30] A. Raval and B. L. Hu, "Backreaction of Hawking Radiation on a Moving Mirror" (1996)

[31] P. Johnson, B. L. Hu and A. Raval, "Backreaction of Unruh Radiation on an Accelerating Detector" (1996)

[32] B. L. Hu, A. Raval and S. Sinha, "Fluctuation-Dissipation Relation for a Radiating Black Hole" (1996)

[33] A. I. Akhiezer and S. V. Peletminsky, Methods of Statistical Physics (Pergamon, London, 1981).

[34] R. Balescu, Equilibrium and Nonequilibrium Statistical Mechanics (John Wiley, New York, 1975)

[35] H. Spohn, Large Scale Dynamics of Interacting Particles (Springer-Verlag, Berlin 1991)

[36] M. Kac and J. Logan, "Fluctuations", in Fluctuation Phenomena, edited by E. W. Montroll and J. L. Lebowitz (Elsevier, New York, 1979), p.1; Phys. Rev. A13, 458 (1976).

[37] S. Nakajima, Progr. Theor. Phys. 20, 948 (1958); R. Zwanzig, J. Chem. Phys. 33, 1338 (1960); and in Lectures in Theoretical Physics III, (ed.) W. E. Britten, B. W. Downes and J. Downs (Interscience, N.Y. 1961) pp. 106-141; H. Mori, Prog. Theor. Phys. 33, 1338 (1965); C. R. Willis and R. H. Picard, Phys. Rev. A9, 1343 (1974); H. Grabert, Projection Operator Techniques in Non Equilibrium Statistical Mechanics (Springer-Verlag, Berlin, 1982).

[38] R. Haag, Local Quantum Physics (Springer, Berlin, 1992).

[39] M. Gell- Mann and J. B. Hartle, Phys. Rev. D47, 3345 (1993). 
[40] B. L. Hu and Y. Zhang, "Coarse-Graining, Scaling, and Inflation" Univ. Maryland Preprint 90-186 (1990); B. L. Hu, in Relativity and Gravitation: Classical and Quantum Proceedings of SILARG VII, Cocoyoc, Mexico, Dec. 1990. eds. J. C. D' Olivo et al (World Scientific, Singapore, 1991).

[41] B. L. Hu, Class. Quan. Grav. 10, S93 (1993)

[42] B. L. Hu and D. J. O'Connor, Phys. Rev. D36, 1701 (1987).

[43] D. N. Page, in Proceedings of the 5th Canadian Conference on General Relativity and Relativistic Astrophysics University of Waterloo, May 1993, eds. R. B. Mann and R. G. McLenaghan (World Scientific, Singapore, 1994) hep-th/9305040

[44] J. D. Bekenstein, in Proceedings of the 7th Marcel Grossmann Meeting on Recent Developments of General Relativity Stanford University, July 1994 eds. R. Ruffini (World Scientific, Singapore 1995) gr-qc/9409015

[45] S. B. Giddings, Lectures at the 1994 Trieste Summer School in High Energy Physics and Cosmology, hep-th/9412138; L. Thorlacius, Lectures at the 1994 Trieste Spring School on String Theory, Gauge Theory and Quantum Gravity, hep-th/9411020

[46] C. Holzhey and F. Wilczek, Nucl. Phys. B380, 447 (1992); F. Wilczek, in Black Holes, Membranes, Wormholes and Superstrings, eds. K. Kalara and D. V. Nanopoulos (World Scientific, Singapore, 1993)

[47] J. R. Anglin, R. Laflamme, W. H. Zurek and J. P. Paz, Phys. Rev. D52, 2221 (1995) 